\title{
ON THE ORBITAL STABILITY FOR A CLASS OF NONAUTONOMOUS NLS
}

\author{
JACOPO BELLAZZINI $^{1}$ AND NICOLA VISCIGLIA ${ }^{2}$ \\ ${ }^{1}$ Dipartimento di Matematica Applicata \\ Università di Pisa \\ Via Buonarroti 1/C, 56127 PISA -Italy \\ 2 Dipartimento di Matematica \\ Università di Pisa \\ Largo B. Pontecorvo 5, 56127 PISA - Italy
}

\begin{abstract}
Following the original approach introduced by T. Cazenave and P.L. Lions in [4] we prove the existence and the orbital stability of standing waves for the following class of NLS:

$$
i \partial_{t} u+\Delta u-V(x) u+Q(x) u|u|^{p-2}=0,(t, x) \in \mathbb{R} \times \mathbb{R}^{n}, 2<p<2+\frac{4}{n}
$$

and

$$
i \partial_{t} u-\Delta^{2} u-V(x) u+Q(x) u|u|^{p-2}=0,(t, x) \in \mathbb{R} \times \mathbb{R}^{n}, 2<p<2+\frac{8}{n}
$$

under suitable assumptions on the potentials $V(x)$ and $Q(x)$. More precisely we assume $V(x), Q(x) \in L^{\infty}\left(\mathbb{R}^{n}\right)$ and meas $\left\{Q(x)>\lambda_{0}\right\} \in(0, \infty)$ for a suitable $\lambda_{0}>0$. The main point is the analysis of the compactness of minimiziang sequences to suitable constrained minimization problems related to $(0.1)$ and $(0.2)$.
\end{abstract}

\section{INTRODUCTION}

The aim of this paper is to prove the existence and the orbital stability (see Definition 1.2) of standing waves for a class of Schrödinger equations with variable coefficients and with a principal part that involves both the laplacian operator $\Delta$ and the bilaplacian operator $\Delta^{2}$. More precisely the model equations that we consider are of the following type:

$$
i \partial_{t} u+\Delta u-V(x) u+Q(x) u|u|^{p-2}=0,(t, x) \in \mathbb{R} \times \mathbb{R}^{n}, 2<p<2+\frac{4}{n}
$$

and

$$
i \partial_{t} u-\Delta^{2} u-V(x) u+Q(x) u|u|^{p-2}=0,(t, x) \in \mathbb{R} \times \mathbb{R}^{n}, 2<p<2+\frac{8}{n}
$$

under suitable assumptions on $V(x)$ and $Q(x)$ that will be specified in the sequel. We recall that a standing wave solution for (1.1) (resp.: (1.2)), is a solution of (1.1) (resp.: (1.2)) of the following type:

$$
u(t, x) \equiv u_{0}(x) e^{i \omega t} \text { with } u_{0} \in H^{1}\left(\mathbb{R}^{n}\right)\left(\text { resp.: } u_{0} \in H^{2}\left(\mathbb{R}^{n}\right)\right) .
$$


In particular $u_{0}(x)$ is solution to one of the following elliptic equations respectively:

$$
\Delta u_{0}-V(x) u_{0}+Q(x) u_{0}|u|_{0}^{p-2}=\omega u_{0}
$$

and

$$
\Delta^{2} u_{0}+V(x) u_{0}-Q(x) u_{0}\left|u_{0}\right|^{p-2}=-\omega u_{0}
$$

for a suitable $\omega \in \mathbb{R}$. We underline that there is an huge literature devoted to the proof of the existence of standing waves to (1.1) and (1.2) (see remarks 1.2 and 1.6) under suitable assumptions on $V(x)$ and $Q(x)$. However the main point in this paper is that, following the general approach introduced in [4], we deduce both the existence and the orbital stability of standing waves.

We recall that in [4] it is proved the existence and the orbital stability of solitary waves for a large class of NLS with constant coefficients, by analysing the compactness of the minimizing sequences of suitable minimization problems. For instance in [4] the existence and the orbital stability of standing waves to (1.1) with $V \equiv 0$, $Q \equiv 1$ and $2<p<2+\frac{4}{n}$ is deduced by looking at the following minimization problem:

$$
\inf _{\mathcal{M}}\left(\frac{1}{2} \int_{\mathbb{R}^{n}}|\nabla u|^{2} d x-\frac{1}{p} \int_{\mathbb{R}^{n}}|u|^{p} d x\right), 2<p<2+\frac{4}{n}
$$

where

$$
\mathcal{M} \equiv\left\{\left.u \in H^{1}\left(\mathbb{R}^{n}\right)\left|\int_{\mathbb{R}^{n}}\right| u\right|^{2} d x=1\right\} .
$$

Notice that the energy and the constraint involved in the minimization problem (1.5) are both quantities preserved along the evolution associated to NLS. More precisely the following conservation laws occur:

$$
E(u(t, x)) \equiv \frac{1}{2} \int_{\mathbb{R}^{n}}|\nabla u(t, x)|^{2} d x-\frac{1}{p} \int_{\mathbb{R}^{n}}|u(t, x)|^{p} d x \equiv E(u(0, x))
$$

and

$$
\int_{\mathbb{R}^{n}}|u(t, x)|^{2} d x \equiv \int_{\mathbb{R}^{n}}|u(0, x)|^{2} d x
$$

where $u(t, x)$ denotes any finite energy solution to (1.1) with $V(x) \equiv 0$ and $Q(x) \equiv$ 1. Due to this fact in [4] the authors have been able to deduce that the set of minimizers to (1.5) are orbitally stables for the flow associated to (1.1) with $V(x) \equiv$ 0 and $Q(x) \equiv 1$, provided that any minimizing sequence for (1.5) is compact in $H^{1}\left(\mathbb{R}^{n}\right)$ up to the action of the translations. Notice that already the proof of the existence of at least a minimizer for (1.5) is not a trivial matter. In fact the problem is translation invariant and hence there is an evident lack of compactness. However this difficulty has been overcomed in [4] by using the concentration-compactness principle (see [8]).

In this paper, following the general approach introduced in [4], we deduce the existence and the orbital stability of standing waves to (1.1) and (1.2) by minimizing the natural energies associated to those equations on the constrained manifold $\|u\|_{L^{2}}=\rho$ for suitable $\rho>0$. More precisely we consider the following minimization problems:

$$
\inf _{H^{1}\left(\mathbb{R}^{n}\right) \cap B_{L^{2}}(\rho)}\left(\frac{1}{2} \int_{\mathbb{R}^{n}}|\nabla u|^{2} d x+\frac{1}{2} \int_{\mathbb{R}^{n}}|u|^{2} V(x) d x-\frac{1}{p} \int_{\mathbb{R}^{n}}|u|^{p} Q(x) d x\right)
$$


and

$$
\inf _{H^{2}\left(\mathbb{R}^{n}\right) \cap B_{L^{2}}(\rho)}\left(\frac{1}{2} \int_{\mathbb{R}^{n}}|\Delta u|^{2} d x+\frac{1}{2} \int_{\mathbb{R}^{n}}|u|^{2} V(x) d x-\frac{1}{p} \int_{\mathbb{R}^{n}}|u|^{p} Q(x) d x\right)
$$

where

$$
B_{L^{2}}(\rho) \equiv\left\{\left.u \in L^{2}\left(\mathbb{R}^{n}\right)\left|\int_{\mathbb{R}^{n}}\right| u\right|^{2} d x=\rho^{2}\right\} .
$$

Let us underline that following the same argument as in [4] one can deduce that the set of minimizers to (1.6) (resp.: (1.7)) is orbitally stables (see Definition 1.2) provided that any minimizing sequence to (1.6) (resp.: (1.7)) is compact in $H^{1}\left(\mathbb{R}^{n}\right)$ (resp.: $H^{2}\left(\mathbb{R}^{n}\right)$ ). We skip the proof of this implication, since it is essentially contained in [4]. Hence, since now on we shall be mainly concentrated on the analysis of the minimizing sequences to (1.6) and (1.7).

Notice that the main difficulty related to the minimization problems (1.6) and (1.7) is connected with the lack of compactness of the Sobolev embedding $H^{s}\left(\mathbb{R}^{n}\right)$ in the corresponding $L^{q}\left(\mathbb{R}^{n}\right)$ spaces. Moreover, due to the presence of the coefficients $V(x)$ and $Q(x)$ in (1.6) and (1.7), our minimization problems are not invariant by the action of the translations, despite to the problems studied in [4]. In order to overcome those difficulties we state below an abstract variational principle that guarantees the compactness of minimizing sequences for a general family of minimization problems.

In the sequel we need the following

Definition 1.1. Let $\mathcal{H}$ be an Hilbert space and $T: \mathcal{H} \rightarrow \mathbb{R}$ a nonlinear map. We say that $T$ is weakly continuous if for every sequence $h_{k} \in \mathcal{H}$ the following implication is satisfied:

$$
h_{k} \rightarrow \bar{h} \text { in } \mathcal{H} \Rightarrow \lim _{k \rightarrow \infty} T\left(h_{k}\right)=T(\bar{h}) .
$$

Proposition 1.1. Let $(\mathcal{H},\|\cdot\|),\left(\mathcal{H}_{1},\|\cdot\|_{1}\right),\left(\mathcal{H}_{2},\|\cdot\|_{2}\right)$ be three Hilbert spaces such that:

$$
\mathcal{H} \subset \mathcal{H}_{1}, \mathcal{H} \subset \mathcal{H}_{2}
$$

and

$$
c\left(\|h\|_{\mathcal{H}_{1}}^{2}+\|h\|_{\mathcal{H}_{2}}^{2}\right) \leq\|h\|_{\mathcal{H}}^{2} \leq C\left(\|h\|_{\mathcal{H}_{1}}^{2}+\|h\|_{\mathcal{H}_{2}}^{2}\right) \forall h \in \mathcal{H}
$$

Assume also that are given $\rho>0$ and two functionals $S, T: \mathcal{H} \rightarrow \mathbb{R}$ such that:

$$
T(0)=0
$$

$T$ is weakly continuous;

$$
T(\lambda h) \leq \lambda^{2} T(h) \text { and } S(\lambda h) \leq \lambda^{2} S(h) \forall \lambda \geq 1, h \in \mathcal{H}
$$

$$
\begin{gathered}
\text { if } h_{k} \rightarrow \bar{h} \text { in } \mathcal{H} \text { and } h_{k} \rightarrow \bar{h} \text { in } \mathcal{H}_{2} \\
\text { then } S\left(h_{k}\right) \rightarrow S(\bar{h}) ;
\end{gathered}
$$

$$
\text { if } h_{k} \rightarrow \bar{h} \text { in } \mathcal{H} \text { then } S\left(h_{k}-\bar{h}\right)+S(\bar{h})=S\left(h_{k}\right)+o(1) \text {; }
$$




$$
-\infty<I_{S, T}^{\rho}<I_{S}^{\rho}
$$

where

and

$$
\begin{gathered}
I_{S, T}^{\rho} \equiv \inf _{h \in B_{\mathcal{H}_{2}}(\rho) \cap \mathcal{H}}\left(\frac{1}{2}\|h\|_{\mathcal{H}_{1}}^{2}+S(h)+T(h)\right) \\
I_{S}^{\rho} \equiv \inf _{h \in B_{\mathcal{H}_{2}}(\rho) \cap \mathcal{H}}\left(\frac{1}{2}\|h\|_{\mathcal{H}_{1}}^{2}+S(h)\right)
\end{gathered}
$$

$$
B_{\mathcal{H}_{2}}(\rho) \equiv\left\{h \in \mathcal{H}_{2} \mid\|h\|_{\mathcal{H}_{2}}=\rho\right\}
$$

$$
\begin{aligned}
& \text { for every sequence }\left\{h_{k}\right\} \in B_{\mathcal{H}_{2}}(\rho) \cap \mathcal{H} \text { such that }\left\|h_{k}\right\|_{\mathcal{H}} \rightarrow \infty, \\
& \text { we have } \frac{1}{2}\left\|h_{k}\right\|_{\mathcal{H}_{1}}^{2}+S\left(h_{k}\right)+T\left(h_{k}\right) \rightarrow \infty \text { as } k \rightarrow \infty .
\end{aligned}
$$

Then every minimizing sequence for (1.14), i.e.

$$
h_{k} \in B_{\mathcal{H}_{2}}(\rho) \cap \mathcal{H} \text { and } \frac{1}{2}\left\|h_{k}\right\|_{\mathcal{H}_{1}}^{2}+S\left(h_{k}\right)+T\left(h_{k}\right) \rightarrow I_{S, T}^{\rho},
$$

is compact in $\mathcal{H}$.

Remark 1.1. There are many concrete situations in which all the assumptions of Proposition 1.1 are satisfied (see for example the proof of Theorems 1.1 and 1.2). Next we show shortly how the existence of a minimizer for (1.5) can be deduced by using Proposition 1.1. In fact due to a symmetrization argument it is sufficient to restrict (1.5) on the space $H_{\text {rad }}^{1}\left(\mathbb{R}^{n}\right)$ of the radially symmetric functions belonging to $H^{1}\left(\mathbb{R}^{n}\right)$. Next we make the following concrete choice of the spaces: $\mathcal{H} \equiv H_{\text {rad }}^{1}\left(\mathbb{R}^{n}\right)$, $\mathcal{H}_{1} \equiv \mathcal{D}_{\text {rad }}^{1,2}\left(\mathbb{R}^{n}\right)$ and $\mathcal{H}_{2} \equiv L_{\text {rad }}^{2}\left(\mathbb{R}^{n}\right)$, where $\mathcal{D}_{\text {rad }}^{1,2}\left(\mathbb{R}^{n}\right)$ and $L_{\text {rad }}^{2}\left(\mathbb{R}^{n}\right)$ denote the radially symmetric functions belonging to the classical Beppo Levi space $\mathcal{D}^{1,2}\left(\mathbb{R}^{n}\right)$ and to the Lebesgue space $L^{2}\left(\mathbb{R}^{n}\right)$ respectively. Finally as functionals $S$ and $T$ we choose respectively $S \equiv 0$ (i.e. the trivial operator) and $T(u)=-\frac{1}{p} \int_{\mathbb{R}^{n}}|u|^{p} d x$. It is easy to check that in this specific framework all the hypothesis of Proposition 1.1 are satisfied. In particular it is easy to check (1.8), (1.10), (1.11), (1.12) while condition (1.9) follows from the compactness of the embedding $H_{\text {rad }}^{1}\left(\mathbb{R}^{n}\right) \subset L^{p}\left(\mathbb{R}^{n}\right)$. By combining an elementary convexity argument with the Sobolev embedding it is easy to show that the l.h.s inequality in (1.13) and (1.15) are satisfied. Finally notice that the r.h.s. inequality in (1.13) in this specific framework becomes $I<0$, where $I$ is the infimum defined in (1.5). The proof of this inequality follows from an elementary rescaling argument.

In order to state our main results it is necessary to give the precise definition of orbital stability (see [4]) that we state below for completeness. First we introduce for every $V(x), Q(x) \in L^{\infty}\left(\mathbb{R}^{n}\right)$ the following quantities:

$$
=\inf _{H^{1}\left(\mathbb{R}^{n}\right) \cap B_{L^{2}}(\rho)}\left(\frac{1}{2} \int_{\mathbb{R}^{n}}|\nabla u|^{2} d x+\frac{1}{2} \int_{\mathbb{R}^{n}}|u|^{2} V(x) d x-\frac{1}{p} \int_{\mathbb{R}^{n}}|u|^{p} Q(x) d x\right)
$$

and

$$
J_{\rho, p}^{V, Q}
$$




$$
=\inf _{H^{2}\left(\mathbb{R}^{n}\right) \cap B_{L^{2}}(\rho)}\left(\frac{1}{2} \int_{\mathbb{R}^{n}}|\Delta u|^{2} d x+\frac{1}{2} \int_{\mathbb{R}^{n}}|u|^{2} V(x) d x-\frac{1}{p} \int_{\mathbb{R}^{n}}|u|^{p} Q(x) d x\right)
$$

where

$$
B_{L^{2}}(\rho) \equiv\left\{\left.u \in L^{2}\left(\mathbb{R}^{n}\right)\left|\int_{\mathbb{R}^{n}}\right| u\right|^{2} d x=\rho^{2}\right\} .
$$

Definition 1.2. The set of minimizers

$$
\mathcal{M}_{\rho, p}^{V, Q} \equiv\left\{u \in H^{1}\left(\mathbb{R}^{n}\right) \cap B_{L^{2}}(\rho) \mid u \text { is minimizer for } I_{\rho, p}^{V, Q}\right\}
$$

(resp.: $\mathcal{N}_{\rho, p}^{V, Q} \equiv\left\{u \in H^{2}\left(\mathbb{R}^{n}\right) \cap B_{L^{2}}(\rho) \mid u\right.$ is any minimizer for $\left.J_{\rho, p}^{V, Q}\right\}$ )

is orbitally stable for (1.1) (resp.: (1.2)) provided that:

$$
\forall \varepsilon, \exists \delta>0 \text { s.t. } \forall v \in H^{1}\left(\mathbb{R}^{N}\right)\left(\text { resp.: } H^{2}\left(\mathbb{R}^{n}\right)\right)
$$

with $\inf _{u \in \mathcal{M}_{\rho, p}^{V, Q}}\|v-u\|_{H^{1}\left(\mathbb{R}^{n}\right)}<\delta\left(\operatorname{resp} .: \inf _{u \in \mathcal{M}_{\rho, p}^{V, Q}}\|v-u\|_{H^{2}\left(\mathbb{R}^{n}\right)}<\delta\right)$, then

$$
\forall t \geq 0 \inf _{u \in \mathcal{M}_{\rho, p}^{V, Q}}\|v(t, \cdot)-u\|_{H^{1}\left(\mathbb{R}^{n}\right)}<\varepsilon\left(\text { resp.: } \inf _{u \in \mathcal{N}_{\rho, p}^{V, Q}}\|v(t, \cdot)-u\|_{H^{2}\left(\mathbb{R}^{n}\right)}<\varepsilon\right)
$$

where $v(t, x)$ is the solution of (1.1) (resp. (1.2)) with initial data $v(x)$.

As a consequence of Proposition 1.1 we prove Theorem 1.1 that mainly concerns the compactness of any minimizing sequence to (1.6) under suitable assumptions on $V(x)$ and $Q(x)$. In this way, by using the general argument in [4] already mentioned above, we get the orbital stability of the set $\mathcal{M}_{\rho, p}^{V, Q}$ introduced in Definition 1.2. In the sequel we denote by meas $(A)$ the Lebesgue measure of the measurable set $A$.

Theorem 1.1. Let $2<p<2+\frac{4}{n}$ and $V(x), Q(x) \in L^{\infty}\left(\mathbb{R}^{n}\right)$. Assume that:

$$
Q(x) \geq 0 \text { a.e. } x \in \mathbb{R}^{n} \text {; }
$$

$$
\text { there is } \lambda_{0}>0 \text { s.t. } 0<\operatorname{meas}\left\{Q(x)>\lambda_{0}\right\}<\infty .
$$

Then there exists $\rho_{0}>0$ such that all the minimizing sequences for (1.16) are compact in $H^{1}\left(\mathbb{R}^{n}\right)$ provided that $\rho>\rho_{0}$. In particular $\mathcal{M}_{\rho, p}^{V, Q}$ is a no empty compact set and it is orbitally stable.

Remark 1.2. Let us underline that the existence of standing waves for (1.1) has been extensively studied in the literature. We mention in particular [3], [7], [8], [11] where existence results are proved under quite general assumptions on $V(x)$, $Q(x)$ and under the following stronger assumption $2<p<\frac{2 n}{n-2}$. However the main point in Theorem 1.1 is that we prove the existence of standing waves by looking at the minimization problem (1.16) and hence we also deduce, following [4], their orbital stability.

Remark 1.3. Notice that, since the solutions constructed in Theorem 1.1 are orbitally stables, it is quite natural to consider nonlinearities that grow at the rate $2<p<2+\frac{4}{n}$. In fact the value $p=2+\frac{4}{n}$ is the critical power for which the Cauchy problem associated to the focusing NLS is globally well-posed (for more details on this point see the comments in [4]).

Remark 1.4. Concerning the assumption (1.18), notice that it is satisfied for instance by any function $Q(x) \in \mathcal{C}\left(\mathbb{R}^{n}\right)$ such that

$$
\sup _{\mathbb{R}^{n}} Q(x)>\limsup _{|x| \rightarrow \infty} Q(x),
$$


which is a condition very much exploited in [7] and [11]. However we underline that in Theorem 1.1 a larger class of functions $Q(x)$ is allowed.

Remark 1.5. We point out that, following the approach in [9] and [10], in Theorem 1.1 are allowed functions $V(x)$ and $Q(x)$ which are not necessarily continuous. As far as we know this is another novelty in this paper compared with previous existence results.

Next we state a version of Theorem 1.1 for equation (1.2). Recall that the set $\mathcal{N}_{\rho, p}^{V, Q}$ has been introduced in Definition 1.2.

Theorem 1.2. Let $2<p<2+\frac{8}{n}$ and $V(x), Q(x) \in L^{\infty}\left(\mathbb{R}^{n}\right)$. Assume that:

$$
Q(x) \geq 0 \text { a.e. } x \in \mathbb{R}^{n} \text {; }
$$

$$
\text { there is } \lambda_{0}>0 \text { s.t. } 0<\operatorname{meas}\left\{Q(x)>\lambda_{0}\right\}<\infty \text {. }
$$

Then there exists $\rho_{0}>0$ such that all the minimizing sequences for (1.17) are compact in $H^{2}\left(\mathbb{R}^{n}\right)$, provided that $\rho>\rho_{0}$. In particular $\mathcal{N}_{\rho, p}^{V, Q}$ is a no empty compact set and it is orbitally stable.

Remark 1.6. Concerning previous existence results of standing waves for (1.2) let us mention [8] where the existence of solutions is proved via the concentrationcompactness argument by assuming that the nonlinear term is regular and has a suitable asymptotic behaviour. In [2] equation (1.4) is treated in the specific case $V(x) \equiv 1$ and with an autonomous nonlinearity $f(u)$. In this case the existence of solutions is proved by means of the compact embedding of $H_{\text {rad }}^{2}\left(\mathbb{R}^{n}\right)$ in $L^{p}\left(\mathbb{R}^{n}\right)$ for $2<p<\frac{2 n}{n-4}$. Many efforts have been devoted to study elliptic equations involving the biharmonic operator with critical nonlinearity $f(u) \equiv u \mid u^{\frac{8}{n-4}}$, see for instance [1] and [5]. Finally let us mention [6] where a deep analysis of the bifurcation properties of the operator $\Delta^{2}-\lambda$ is done.

In the best of our knowledge the existence result stated in Theorem 1.2 under such a general assumptions on $V(x)$ and $Q(x)$ is not written elsewhere. Moreover, since we deduce the existence result in Theorem 1.2 by looking at the minimization problem (1.17) we also get, following [4], the orbital stability of the corresponding solutions. As far as we know the question of the stability of solitary waves to (1.2) has not been analysed in previous papers.

Next we fix some notations. For every $x \in \mathbb{R}^{n}, R>0$ we denote by $B_{R}(x)$ the ball in $\mathbb{R}^{n}$ of radius $R$ and centered in $x$.

For every $1 \leq p \leq \infty$ we denote by $\|\cdot\|_{p}$ the classical $L^{p}\left(\mathbb{R}^{n}\right)$-norm and for every measurable set $A$ we denote by meas $(A)$ its Lebesgue measure.

Given $\rho>0$ we use the notation

$$
B_{L^{2}}(\rho) \equiv\left\{\left.u \in L^{2}\left(\mathbb{R}^{n}\right)\left|\int_{\mathbb{R}^{n}}\right| u\right|^{2} d x=\rho^{2}\right\} .
$$

The paper is organized ad follows: section 2 is devoted to the proof of Proposition 1.1, in sections 3 and 4 we prove respectively Theorem 1.1 and 1.2. In the Appendix we show the existence of a minimizer for $(1.7)$ when $V(x) \equiv 0$ and $Q(x) \equiv 1$. This fact is important along the proof of Theorem 1.2. Let us point out that the content of the Appendix is essentially contained in [8], however for the sake of completeness we give all the details of the proof. 


\section{Proof of Proposition 1.1}

By assumption (1.15) we have that $\sup _{k \in \mathbb{N}}\left\|h_{k}\right\|_{\mathcal{H}}<\infty$ hence we can assume $h_{k} \rightarrow \bar{h}$ in $\mathcal{H}$. On the other hand we have

$$
\left\|h_{k}\right\|_{\mathcal{H}_{2}}=\rho \text { and } \frac{1}{2}\left\|h_{k}\right\|_{\mathcal{H}_{1}}^{2}+S\left(h_{k}\right)+T\left(h_{k}\right)=I_{S, T}^{\rho}+o(1)
$$

and due to assumption (1.9) we get

$$
T\left(h_{k}\right)=T(\bar{h})+o(1)
$$

By combining (2.1) and (2.2) we get:

$$
\frac{1}{2}\left\|h_{k}\right\|_{\mathcal{H}_{1}}^{2}+S\left(h_{k}\right)+T(\bar{h})=I_{S, T}^{\rho}+o(1) .
$$

In particular this implies

$$
I_{S}^{\rho}+T(\bar{h}) \leq I_{S, T}^{\rho}
$$

and hence due to (1.8) and (1.13) we get $\bar{h} \neq 0$.

On the other hand by combining (2.3) with (1.12) we get:

$$
\begin{gathered}
\frac{1}{2}\left\|h_{k}-\bar{h}\right\|_{\mathcal{H}_{1}}^{2}+\frac{1}{2}\|\bar{h}\|_{\mathcal{H}_{1}}^{2}+S\left(h_{k}-\bar{h}\right)+S(\bar{h}) \\
+T(\bar{h})=I_{S, T}^{\rho}+o(1) .
\end{gathered}
$$

Moreover we have

$$
\left\|h_{k}-\bar{h}\right\|_{\mathcal{H}_{2}}^{2}=\left\|h_{k}\right\|_{\mathcal{H}_{2}}^{2}-\|\bar{h}\|_{\mathcal{H}_{2}}^{2}+o(1)=\rho^{2}-\|\bar{h}\|_{\mathcal{H}_{2}}^{2}+o(1)
$$

and hence, since $\bar{h} \neq 0$, we deduce that

$$
\left\|h_{k}-\bar{h}\right\|_{\mathcal{H}_{2}}^{2}<\rho^{2}
$$

provided that $k$ is large enough. Next notice that since $\bar{h}$ is the weak limit of the sequence $\left\{h_{k}\right\}$ and since $\left\|h_{k}\right\|_{\mathcal{H}_{2}}=\rho$, we get

$$
\|\bar{h}\|_{\mathcal{H}_{2}} \leq \rho .
$$

By combining (2.4), (2.6), (2.7) with the assumption (1.10) we deduce

$$
\begin{gathered}
\frac{\left\|h_{k}-\bar{h}\right\|_{\mathcal{H}_{2}}^{2}}{\rho^{2}}\left(\frac{1}{2}\left\|\frac{\rho\left(h_{k}-\bar{h}\right)}{\left\|h_{k}-\bar{h}\right\|_{\mathcal{H}_{2}}}\right\|_{\mathcal{H}_{1}}^{2}+S\left(\frac{\rho\left(h_{k}-\bar{h}\right)}{\left\|h_{k}-\bar{h}\right\|_{\mathcal{H}_{2}}}\right)\right) \\
+\frac{\|\bar{h}\|_{\mathcal{H}_{2}}^{2}}{\rho^{2}}\left(\frac{1}{2}\left\|\frac{\rho \bar{h}}{\|\bar{h}\|_{\mathcal{H}_{2}}}\right\|_{\mathcal{H}_{1}}^{2}+T\left(\frac{\rho \bar{h}}{\|\bar{h}\|_{\mathcal{H}_{2}}}\right)+S\left(\frac{\rho \bar{h}}{\|\bar{h}\|_{\mathcal{H}_{2}}}\right)\right) \\
\leq I_{S, T}^{\rho}+o(1)
\end{gathered}
$$

and hence due to (2.5)

$$
\begin{gathered}
\frac{1}{\rho^{2}}\left(\left\|h_{k}-\bar{h}\right\|_{\mathcal{H}_{2}}^{2} I_{S}^{\rho}+\|\bar{h}\|_{\mathcal{H}_{2}}^{2} I_{S, T}^{\rho}\right) \\
\leq I_{S, T}^{\rho} \frac{1}{\rho^{2}}\left(\|\bar{h}\|_{\mathcal{H}_{2}}^{2}+\left\|h_{k}-\bar{h}\right\|_{\mathcal{H}_{2}}^{2}\right)+o(1),
\end{gathered}
$$

that in turn implies:

$$
\left\|h_{k}-\bar{h}\right\|_{\mathcal{H}_{2}}^{2}\left(I_{S}^{\rho}-I_{S, T}^{\rho}\right) \leq o(1)
$$

By (1.13) we get $h_{k} \rightarrow \bar{h}$ strongly in $\mathcal{H}_{2}$. It is now easy to deduce by (1.11) that the convergence occurs strongly also in $\mathcal{H}$. 


\section{Applications to NLS}

Given $\rho>0, p>2$ and $U(x), W(x) \in L^{\infty}\left(\mathbb{R}^{n}\right)$ we define the following quantities:

$$
I_{\rho, p}^{U, W}=\inf _{H^{1}\left(\mathbb{R}^{n}\right) \cap B_{L^{2}}(\rho)}\left(\frac{1}{2}\|\nabla u\|_{2}^{2}+\frac{1}{2} \int_{\mathbb{R}^{n}}|u|^{2} U(x) d x-\frac{1}{p} \int_{\mathbb{R}^{n}}|u|^{p} W(x) d x\right) .
$$

Moreover if $V(x) \in L^{\infty}\left(\mathbb{R}^{n}\right)$ is as in Theorem 1.1, then we introduce the new function

$$
\tilde{V}(x) \equiv V(x)-\operatorname{infess}_{\mathbb{R}^{n}} V(x) .
$$

Notice that $\tilde{V}$ is a non-negative function, i.e. $\tilde{V}(x) \geq 0$ a.e. $x \in \mathbb{R}^{n}$. This property will be important along the proof of next Lemma.

Lemma 3.1. Assume that $V(x), Q(x), \lambda_{0}$ and $p$ are as in Theorem 1.1. Then there exists $\rho_{0}>0$ such that:

$$
I_{\rho, p}^{\tilde{V}, Q}<I_{\rho, p}^{\tilde{V}, M i n}\left\{Q, \lambda_{0}\right\} \forall \rho>\rho_{0} .
$$

Proof. Since $Q(x) \in L^{\infty}\left(\mathbb{R}^{n}\right)$ we deduce by the Lebesgue derivation Theorem that:

$$
\lim _{\delta \rightarrow 0} \delta^{-n} \int_{B_{\delta}\left(x_{0}\right)}\left|Q(x)-Q\left(x_{0}\right)\right| d x=0 \text { a.e. } x_{0} \in \mathbb{R}^{n} .
$$

Since by assumption we have meas $\left\{x \in \mathbb{R}^{n} \mid Q(x)>\lambda_{0}\right\}>0$, we deduce that there exists $\bar{x} \in\left\{x \in \mathbb{R}^{n} \mid Q(x)>\lambda_{0}\right\}$ such that $\lim _{\delta \rightarrow 0} \delta^{-n} \int_{B_{\delta}(\bar{x})}|Q(x)-Q(\bar{x})| d x=0$. For simplicity we can assume that $\bar{x} \equiv 0$ and also $\bar{\lambda} \equiv Q(0)$, hence we have

$$
\lim _{\delta \rightarrow 0} \delta^{-n} \int_{B_{\delta}(0)}|Q(x)-\bar{\lambda}| d x=0 \text { with } \bar{\lambda}>\lambda_{0} .
$$

Next we fix a minimizer $u_{0} \in H^{1}\left(\mathbb{R}^{n}\right)$ for $I_{1, p}^{0, \lambda_{0}}$ (the proof of the existence of a minimizer is given in [8], see also remark 1.1). Then it is easy to check that

$$
u_{0}^{\rho} \equiv u_{0}\left(\frac{x}{\rho^{\alpha}}\right) \rho^{-\beta} \text { is a minimizer for } I_{\rho, p}^{0, \lambda_{0}},
$$

where

$$
\alpha=\alpha(n, p)=\frac{2(p-2)}{(p-2) n-4} \text { and } \beta=\beta(n, p)=\frac{4}{(p-2) n-4} .
$$

We claim that there is $\rho_{0}>0$ such that

$$
I_{\rho, p}^{\tilde{V}, Q}<I_{\rho, p}^{0, \lambda_{0}} \forall \rho>\rho_{0} .
$$

On the other hand $\tilde{V}(x) \geq 0$ and $0 \leq \operatorname{Min}\left\{Q(x), \lambda_{0}\right\} \leq \lambda_{0}$ and this implies

$$
I_{\rho, p}^{0, \lambda_{0}} \leq I_{\rho, p}^{\tilde{V}, M i n}\left\{Q(x), \lambda_{0}\right\} .
$$

By combining (3.5) with (3.6) we deduce the desired result.

Next we prove (3.5). Due to (3.3) it is sufficient to prove the following inequality:

or equivalently

$$
\begin{aligned}
\frac{1}{2}\left\|\nabla u_{0}^{\rho}\right\|_{2}^{2} & +\frac{1}{2} \int_{\mathbb{R}^{n}}\left|u_{0}^{\rho}\right|^{2} \tilde{V}(x) d x-\frac{1}{p} \int_{\mathbb{R}^{n}}\left|u_{0}^{\rho}\right|^{p} Q(x) d x \\
& <\frac{1}{2}\left\|\nabla u_{0}^{\rho}\right\|_{2}^{2}-\frac{\lambda_{0}}{p} \int_{\mathbb{R}^{n}}\left|u_{0}^{\rho}\right|^{p} d x
\end{aligned}
$$

$$
I(\rho)+I I(\rho)+I I I(\rho)
$$




$$
\equiv \frac{1}{2} \int_{\mathbb{R}^{n}}\left|u_{0}^{\rho}\right|^{2} \tilde{V}(x) d x-\frac{1}{p} \int_{\mathbb{R}^{n}}\left|u_{0}^{\rho}\right|^{p}(Q(x)-\bar{\lambda}) d x+\frac{\lambda_{0}-\bar{\lambda}}{p} \int_{\mathbb{R}^{n}}\left|u_{0}^{\rho}\right|^{p} d x<0 .
$$

Let us fix $R_{0}>0$ such that

$$
\frac{2}{p}\|Q\|_{\infty} \int_{|x|>R_{0}}\left|u_{0}\right|^{p} d x+\frac{\lambda_{0}-\bar{\lambda}}{p}\left\|u_{0}\right\|_{p}^{p}=-\epsilon_{0}<0
$$

and notice that due to (3.2) we get:

$$
\begin{gathered}
I(\rho) \leq \frac{1}{2}\|\tilde{V}\|_{\infty}\left\|u_{0}^{\rho}\right\|_{2}^{2}=\frac{1}{2}\|\tilde{V}\|_{\infty} \rho^{2} ; \\
I I(\rho) \leq \frac{1}{p} \rho^{-p \beta}\left\|u_{0}\right\|_{\infty}^{p} \int_{B\left(0, R_{0} \rho^{\alpha}\right)}|Q(x)-\bar{\lambda}| d x+\frac{2}{p}\|Q\|_{\infty} \int_{|x|>R_{0} \rho^{\alpha}}\left|u_{0}^{\rho}\right|^{p} d x \\
=o(1) \rho^{n \alpha-p \beta}+\frac{2}{p}\|Q\|_{\infty} \rho^{(n \alpha-p \beta)} \int_{|x|>R_{0}}\left|u_{0}\right|^{p} d x
\end{gathered}
$$

where $\lim _{\rho \rightarrow \infty} o(1)=0$;

$$
I I I(\rho)=\frac{\left(\lambda_{0}-\bar{\lambda}\right)}{p} \int_{\mathbb{R}^{n}}\left|u_{0}^{\rho}\right|^{p} d x=\frac{\left(\lambda_{0}-\bar{\lambda}\right)}{p} \rho^{n \alpha-p \beta}\left\|u_{0}\right\|_{p}^{p} .
$$

Hence we finally get

$$
\begin{gathered}
I(\rho)+I I(\rho)+\operatorname{III}(\rho) \\
\leq \frac{1}{2}\|\tilde{V}\|_{\infty} \rho^{2}-\epsilon_{0} \rho^{n \alpha-p \beta}\left\|u_{0}\right\|_{p}^{p}+o(1) \rho^{n \alpha-p \beta}
\end{gathered}
$$

where $\epsilon_{0}>0$ is the constant that appear in (3.8). Since $2<n \alpha-p \beta$, the inequality (3.9) implies (3.7) for $\rho$ large enough, and in turn it is equivalent to (3.5).

Proof of Theorem 1.1 Notice that by definition of $\tilde{V}$ (see (3.1)) we have $V-\tilde{V} \equiv$ const. Due to this fact it is easy to deduce that the minimizing sequences for $I_{\rho, p}^{V, Q}$ are precisely the minimizing sequences for $I_{\rho, p}^{\tilde{V}, Q}$. Hence it is sufficient to show that any minimizing sequence for $I_{\rho, p}^{\tilde{V}, Q}$ is compact in $H^{1}\left(\mathbb{R}^{n}\right)$ in order to deduce the same property for the minimizing sequences for $I_{\rho, p}^{V, Q}$. In order to prove this fact we use Proposition 1.1 where we make the following specific choice for the spaces $\mathcal{H}, \mathcal{H}_{1}, \mathcal{H}_{2}$ and for the operators $S$ and $T$ :

$$
\begin{gathered}
\mathcal{H}=H^{1}\left(\mathbb{R}^{n}\right), \mathcal{H}_{1}=\mathcal{D}^{1,2}\left(\mathbb{R}^{n}\right), \mathcal{H}_{2}=L^{2}\left(\mathbb{R}^{n}\right), \\
S(u)=\int_{\mathbb{R}^{n}}\left(\frac{1}{2}|u|^{2} \tilde{V}(x)-\frac{1}{p}|u|^{p} \min \left\{Q(x), \lambda_{0}\right\}\right) d x, \\
T(u)=-\frac{1}{p} \int_{Q(x) \geq \lambda_{0}}|u|^{p}\left(Q(x)-\lambda_{0}\right) d x
\end{gathered}
$$

and we also assume $\rho>\rho_{0}$ where $\rho_{0}$ is the same constant that appears in Lemma 3.1. It is easy to verify that in this specific framework the assumptions $(1.8),(1.10)$ in Proposition 1.1 are satisfied. The r.h.s inequality in (1.13) follows from Lemma 3.1 provided that $\rho>\rho_{0},(1.9)$ is a consequence of the Rellich Compactness Theorem in conjunction with hypothesis (1.18). Condition (1.11) comes from the following elementary fact:

$$
\begin{gathered}
\text { if } u_{k} \rightarrow \bar{u} \text { in } L^{2}\left(\mathbb{R}^{n}\right) \text { and } u_{k} \text { is bounded in } H^{1}\left(\mathbb{R}^{n}\right) \\
\text { then } u_{k} \rightarrow \bar{u} \text { in } L^{p}\left(\mathbb{R}^{n}\right) \forall 2 \leq p<\frac{2 n}{n-2} .
\end{gathered}
$$


In order to deduce the l.h.s. inequality (1.13) and (1.15), notice that by combining the convexity inequality with the Sobolev embedding we get:

$$
\|u\|_{p} \leq C\|\nabla u\|_{2}^{\frac{n(p-2)}{2 p}}\|u\|_{2}^{1-\frac{n}{2}+\frac{n}{p}}=C\|\nabla u\|_{2}^{\frac{n(p-2)}{2 p}} \rho^{1-\frac{n}{2}+\frac{n}{p}} \forall u \in H^{1}\left(\mathbb{R}^{n}\right) \cap B_{L^{2}}(\rho)
$$

and this implies

$$
\begin{gathered}
\frac{1}{2}\|\nabla u\|_{2}^{2}+\int_{\mathbb{R}^{n}}|u|^{2} \tilde{V}(x) d x-\int_{\mathbb{R}^{n}}|u|^{p} Q(x) d x \\
\geq \frac{1}{2}\|\nabla u\|_{2}^{2}-C(\rho)\|Q\|_{\infty}\|\nabla u\|_{2}^{\frac{n(p-2)}{2}}
\end{gathered}
$$

where we have used $\tilde{V}(x) \geq 0$. Since we are assuming $2<p<2+\frac{4}{n}$ we have $2>\frac{n(p-2)}{2}$ and hence by (3.10) we can deduce both the l.h.s. inequality in (1.13) and (1.15). Finally we shall check condition (1.12). From the Rellich Compactness Theorem we get:

$$
\text { if } u_{k} \rightarrow \bar{u} \text { in } H^{1}\left(\mathbb{R}^{n}\right) \text { then } u_{k} \rightarrow \bar{u} \text { in } L^{p}(B(0, R)) \forall R>0 .
$$

It is also easy to check that:

$$
\begin{gathered}
\text { if } u_{k} \rightarrow \bar{u} \text { in } H^{1}\left(\mathbb{R}^{n}\right) \text { then } \\
\int_{\mathbb{R}^{n}}\left|u_{k}\right|^{2} \tilde{V}(x) d x=\int_{\mathbb{R}^{n}}\left|u_{k}-\bar{u}\right|^{2} \tilde{V}(x) d x+\int_{\mathbb{R}^{n}}|\bar{u}|^{2} \tilde{V}(x) d x+o(1)
\end{gathered}
$$

hence (1.12) will follow from:

$$
\lim _{k \rightarrow \infty}\left|\int_{\mathbb{R}^{n}}\left(\left|u_{k}\right|^{p}-\left|u_{k}-\bar{u}\right|^{p}-|\bar{u}|^{p}\right) \min \left\{Q(x), \lambda_{0}\right\} d x\right|=0
$$

provided that $u_{k} \rightarrow \bar{u}$ in $H^{1}\left(\mathbb{R}^{n}\right)$. Since $\bar{u} \in L^{p}\left(\mathbb{R}^{n}\right)$ we have:

$$
\lim _{R \rightarrow \infty} \int_{\mathbb{R}^{n} \backslash B(0, R)}|\bar{u}|^{p} \min \left\{Q(x), \lambda_{0}\right\} d x=0 .
$$

On the other hand since $p>2$ we can use the mean value theorem in order to deduce the existence of a constant $C=C(p)>0$ such that

$$
|| t+\left.h\right|^{p}-|t|^{p}|\leq C| h \mid(|t|+|h|)^{p-1} \forall t, h \in \mathbb{R} .
$$

In particular for every $k \in \mathbb{N}$ we have

$$
|| u_{k}(x)-\left.\bar{u}(x)\right|^{p}-\left|u_{k}(x)\right|^{p}|\leq C| \bar{u}(x) \mid\left(\left|u_{k}(x)\right|+|\bar{u}(x)|\right)^{p-1} \text { a.e. } x \in \mathbb{R}^{n}
$$

that by the Hölder inequality implies

$$
\begin{gathered}
\int_{\Omega}\left\|u_{k}(x)-\left.\bar{u}(x)\right|^{p}-\mid u_{k}(x)\right\|^{p} \min \left\{Q(x), \lambda_{0}\right\} d x \\
\leq C\left\|\min \left\{Q(x), \lambda_{0}\right\}\right\|_{L^{\infty}\left(\mathbb{R}^{n}\right)}\|\bar{u}\|_{L^{p}(\Omega)}\left(\left\|\left|u_{k}\right|+|\bar{u}|\right\|_{L^{p}(\Omega)}^{\frac{p-1}{p}}\right)
\end{gathered}
$$

for every measurable set $\Omega \subset \mathbb{R}^{n}$. By combining this estimate (where we choose $\Omega=\mathbb{R}^{n} \backslash B(0, R)$ ) with the uniform boundedness of $\left\|u_{k}\right\|_{L^{p}\left(\mathbb{R}^{n}\right)}$ (that follows from the usual Sobolev embedding $\left.H^{1}\left(\mathbb{R}^{n}\right) \subset L^{p}\left(\mathbb{R}^{n}\right)\right)$ and with (3.13) we get

$$
\begin{aligned}
\forall \epsilon & >0 \exists R(\epsilon)>0 \text { s.t. }|r(k, \epsilon)|<\epsilon \forall k \in \mathbb{N} \text { where } \\
r(k, \epsilon) & \equiv \int_{\mathbb{R}^{n} \backslash B(0, R(\epsilon))}\left(\left|u_{k}-\bar{u}\right|^{p}-\left|u_{k}\right|^{p}\right) \min \left\{Q(x), \lambda_{0}\right\} d x .
\end{aligned}
$$


Moreover due to (3.11) we get

$$
\lim _{k \rightarrow \infty} s(k, \epsilon)=0 \text { and } \lim _{k \rightarrow \infty} t(k, \epsilon)=0 \forall \epsilon>0
$$

where

and

$$
s(k, \epsilon) \equiv \int_{B(0, R(\epsilon))}\left(\left|u_{k}\right|^{p}-|\bar{u}|^{p}\right) \min \left\{Q(x), \lambda_{0}\right\} d x
$$

$$
t(k, \epsilon) \equiv \int_{B(0, R(\epsilon))}\left|u_{k}-\bar{u}\right|^{p} \min \left\{Q(x), \lambda_{0}\right\} d x .
$$

In particular we deduce that there exists $k(\epsilon) \in \mathbb{N}$ such that

$$
|s(k, \epsilon)| \text { and }|t(k, \epsilon)|<\epsilon \forall k>k(\epsilon) .
$$

Moreover we have

$$
\begin{gathered}
\int_{\mathbb{R}^{n}}\left|u_{k}\right|^{p} \min \left\{Q(x), \lambda_{0}\right\} d x \\
=\int_{\mathbb{R}^{n} \backslash B(0, R(\epsilon))}\left|u_{k}\right|^{p} \min \left\{Q(x), \lambda_{0}\right\} d x+\int_{B(0, R(\epsilon))}\left|u_{k}\right|^{p} \min \left\{Q(x), \lambda_{0}\right\} d x \\
=-r(k, \epsilon)+\int_{\mathbb{R}^{n} \backslash B(0, R(\epsilon))}\left|u_{k}-\bar{u}\right|^{p} \min \left\{Q(x), \lambda_{0}\right\} d x \\
+s(k, \epsilon)+\int_{B(0, R(\epsilon))}|\bar{u}|^{p} \min \left\{Q(x), \lambda_{0}\right\} d x \\
=-r(k, \epsilon)-t(k, \epsilon)+\int_{\mathbb{R}^{n}}\left|u_{k}-\bar{u}\right|^{p} \min \left\{Q(x), \lambda_{0}\right\} d x \\
+s(k, \epsilon)+\int_{\mathbb{R}^{n}}|\bar{u}|^{p} \min \left\{Q(x), \lambda_{0}\right\} d x-\int_{\mathbb{R}^{n} \backslash B(0, R(\epsilon))}|\bar{u}|^{p} \min \left\{Q(x), \lambda_{0}\right\} d x .
\end{gathered}
$$

Moreover (3.15) and (3.16) imply

$$
|r(k, \epsilon)|+|s(k, \epsilon)|+|t(k, \epsilon)|<3 \epsilon \forall k>k(\epsilon)
$$

and hence

$$
\begin{aligned}
& \left|\int_{\mathbb{R}^{n}}\left(\left|u_{k}\right|^{p}-\left|u_{k}-\bar{u}\right|^{p}-|\bar{u}|^{p}\right) \min \left\{Q(x), \lambda_{0}\right\} d x\right| \\
< & 3 \epsilon+\int_{\mathbb{R}^{n} \backslash B(0, R(\epsilon))}|\bar{u}|^{p} \min \left\{Q(x), \lambda_{0}\right\} d x \forall k>k(\epsilon) .
\end{aligned}
$$

Due to (3.13) and since $R(\epsilon) \rightarrow \infty$ as $\epsilon \rightarrow 0$ it is now easy to deduce (3.12).

\section{Applications to NLS involving the Biharmonic operator}

The proof of Theorem 1.2 follows the same lines of the proof of Theorem 1.1. A basic tool is the following version of Lemma 3.1 where, given $\rho>0, p>2$ and $U(x), W(x) \in L^{\infty}\left(\mathbb{R}^{n}\right)$, the quantity $J_{\rho, p}^{U, W}$ is defined as follows:

$$
J_{\rho, p}^{U, W}=\inf _{H^{2}\left(\mathbb{R}^{n}\right) \cap B_{L^{2}}(\rho)}\left(\frac{1}{2}\|\Delta u\|_{2}^{2}+\frac{1}{2} \int_{\mathbb{R}^{n}}|u|^{2} U(x) d x-\frac{1}{p} \int_{\mathbb{R}^{n}}|u|^{p} W(x) d x\right) .
$$

In next Lemma the potential $\tilde{V}(x)$ is the one introduced in (3.1). 
Lemma 4.1. Assume that $V(x), Q(x), \lambda_{0}$ and $p$ are as in Theorem 1.2. Then there exists $\rho_{0}>0$ such that

$$
J_{\rho, p}^{\tilde{V}, Q}<J_{\rho, p}^{\tilde{V}, \min }\left\{Q(x), \lambda_{0}\right\} \forall \rho>\rho_{0} .
$$

It is easy to check that the proof of Lemma 3.1 can be adapted in order to prove Lemma 4.1, provided that we are able to show the existence of a minimizer to the following minimization problem:

$$
J_{\rho, p}^{0, \lambda_{0}}=\inf _{H^{2}\left(\mathbb{R}^{n}\right) \cap B_{L^{2}}(\rho)}\left(\frac{1}{2}\|\Delta u\|_{2}^{2}-\frac{\lambda_{0}}{p} \int_{\mathbb{R}^{n}}|u|^{p} d x\right) .
$$

The detailed proof of this fact is given for completeness in the Appendix, even if it is essentially contained in [8]. We skip the detailed proof of Lemma 4.1.

Proof of Theorem 1.2 As in the proof of Theorem 1.1, it sufficient to prove the compactness of the minimizing sequences for $J_{\rho, p}^{\tilde{V}, Q}$. This fact can be shown by using Proposition 1.1 in the following explicit framework:

$$
\begin{gathered}
\mathcal{H}=H^{2}\left(\mathbb{R}^{n}\right), \mathcal{H}_{1}=\mathcal{D}^{2,2}\left(\mathbb{R}^{n}\right), \mathcal{H}_{2}=L^{2}\left(\mathbb{R}^{n}\right), \\
S(u)=\int\left(\frac{1}{2}|u|^{2} \tilde{V}(x)-\frac{1}{p}|u|^{p} \min \left\{Q(x), \lambda_{0}\right\}\right) d x, \\
\left.T(u)=-\frac{1}{p} \int_{Q(x) \geq \lambda_{0}}|u|^{p}\left(Q(x)-\lambda_{0}\right)\right) d x
\end{gathered}
$$

and $\rho>\rho_{0}$ where $\rho_{0}$ is the constant that appears in Lemma 4.1. It is easy to check, following the same arguments involved in the proof of Theorem 1.1, that all the assumptions required in Proposition 1.1 are satisfied in this specific context. In particular the r.h.s inequality in (1.13) follows from Lemma 4.1. However we skip the details of the proof.

\section{Appendix}

The aim of this Appendix is to give a detailed proof of Theorem 5.1. We recall that it is essentially contained in [8] however, since it plays a fundamental role along the proof of Theorem 1.2, we give all the details of the proof for completeness.

In the sequel we denote by $Q_{R}(y)$ the cube

$$
\left[y_{1}-\frac{R}{2}, y_{1}+\frac{R}{2}\right) \times \ldots \times\left[y_{n}-\frac{R}{2}, y_{n}+\frac{R}{2}\right)
$$

where $y \equiv\left(y_{1}, \ldots ., y_{n}\right) \in \mathbb{R}^{n}$ and $R>0$.

Theorem 5.1. For every $\rho>0$ and for every $2<p<2+\frac{8}{n}$, there is a minimizer for the following problem:

$$
J_{\rho, p}=\inf _{H^{2}\left(\mathbb{R}^{n}\right) \cap B_{L^{2}}(\rho)}\left(\frac{1}{2}\|\Delta u\|_{2}^{2}-\frac{1}{p} \int_{\mathbb{R}^{n}}|u|^{p} d x\right) .
$$

Remark 5.1. In fact we prove a stronger version of Theorem 5.1. More precisely we show that all the minimizing sequences for $J_{\rho, p}$ are compact in $H^{2}\left(\mathbb{R}^{n}\right)$ up to the action of the translations.

In the sequel we need the following

Proposition 5.1. If $2<p<2+\frac{8}{n}$ and $\rho>0$, then: 
- $J_{\rho, p}>-\infty$;

- any minimizing sequence $u_{k} \in H^{2}\left(\mathbb{R}^{n}\right) \cap B_{L^{2}}(\rho)$ for (5.1) is bounded in $H^{2}\left(\mathbb{R}^{n}\right)$

- $J_{\rho, p}<0$.

Proof By combining the Sobolev embedding $\|u\|_{\frac{2 n}{n-4}} \leq C\|\Delta u\|_{2}$ with the convexity inequality we get:

$$
\|u\|_{p} \leq C\|u\|_{2}^{\frac{2 n-n p+4 p}{4 p}}\|\Delta u\|_{2}^{\frac{n(p-2)}{4 p}} .
$$

From this inequality it is easy to deduce that the functional

$$
H^{2}\left(\mathbb{R}^{n}\right) \cap B_{L^{2}}(\rho) \ni u \rightarrow \frac{1}{2}\|\Delta u\|_{2}^{2}-\frac{1}{p} \int_{\mathbb{R}^{n}}|u|^{p} d x
$$

is bounded from below and moreover every minimizing sequence is bounded in $H^{2}\left(\mathbb{R}^{n}\right)$, provided that $2<p<2+\frac{8}{n}$.

Finally we shall prove that $J_{\rho, p}<0$. We fix $v \in H^{2}\left(\mathbb{R}^{n}\right) \cap B_{L^{2}}(\rho)$, then $v_{\lambda} \equiv$ $\lambda^{-\frac{n}{2}} v\left(\frac{x}{\lambda}\right)$ belongs to $H^{2}\left(\mathbb{R}^{n}\right) \cap B_{L^{2}}(\rho)$. Moreover we have

$$
\begin{gathered}
J_{\rho, p} \leq \frac{1}{2}\left\|\Delta v_{\lambda}\right\|_{2}^{2}-\frac{1}{p} \int_{\mathbb{R}^{n}}\left|v_{\lambda}\right|^{p} d x \\
=\frac{1}{2 \lambda^{4}} \int_{\mathbb{R}^{n}}|\Delta v|^{2} d x-\frac{\lambda^{n\left(1-\frac{p}{2}\right)}}{p} \int_{\mathbb{R}^{n}}|v|^{p} d x \forall \lambda>0
\end{gathered}
$$

and this implies $J_{\rho, p}<0$ provided that we choose $\lambda$ in a suitable way.

Proposition 5.2. Let $u_{k}$ is a sequence bounded in $H^{2}\left(\mathbb{R}^{n}\right)$ and such that

$$
\lim _{k \rightarrow \infty}\left(\sup _{y \in \mathbb{R}^{n}} \int_{Q_{1}(y)}\left|u_{k}\right|^{2} d x\right)=0 .
$$

Then $u_{k} \rightarrow 0$ in $L^{q}\left(\mathbb{R}^{n}\right)$ for any $q \in\left(2, \frac{2 N}{N-4}\right)$.

Proof By the convexity inequality we get

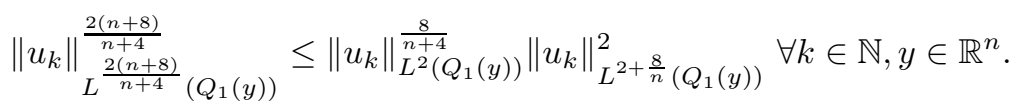

Next we introduce for every $z \equiv\left(z_{1}, \ldots, z_{n}\right) \in \mathbb{Z}^{n}$ the cube

$$
Q_{z} \equiv\left[z_{1}, z_{1}+1\right) \times \ldots \times\left[z_{n}, z_{n}+1\right) .
$$

Notice that $\mathbb{R}^{n}$ is the disjoint union of the cubes $Q_{z}$ with $z \in \mathbb{Z}^{n}$. Hence we get

$$
\begin{gathered}
\left\|u_{k}\right\|_{\frac{2(n+8)}{n+4}}^{\frac{2(n+8)}{n+4}}=\sum_{z \in \mathbb{Z}}\left\|u_{k}\right\|_{L^{\frac{2(n+8)}{n+4}}\left(Q_{z}\right)}^{\frac{2(n+8)}{n+4}} \\
\leq \sum_{z \in \mathbb{Z}^{n}}\left\|u_{k}\right\|_{L^{2}\left(Q_{z}\right)}^{\frac{8}{n+4}}\|u\|_{L^{2+\frac{8}{n}}\left(Q_{z}\right)}^{2} \leq C\left(\sup _{y \in \mathbb{R}^{n}} \int_{Q_{1}(y)}\left|u_{k}\right|^{2} d x\right)^{\frac{4}{n+4}} \sum_{z \in \mathbb{Z}}\left\|u_{k}\right\|_{H^{2}\left(Q_{z}\right)}^{2} \\
=\left(\sup _{y \in \mathbb{R}^{n}} \int_{Q_{1}(y)}\left|u_{k}\right|^{2} d x\right)^{\frac{4}{n+4}}\left\|u_{k}\right\|_{H^{2}\left(\mathbb{R}^{n}\right)}^{2}
\end{gathered}
$$


where we have the Sobolev embedding $H^{2}\left(Q_{z}\right) \subset L^{2+\frac{8}{n}}\left(Q_{z}\right)$. By using the assumptions on $u_{k}$ we finally deduce:

$$
\lim _{k \rightarrow \infty}\left\|u_{k}\right\|_{\frac{2(n+8)}{n+4}}=0 .
$$

By combining this fact, with the embedding

$$
H^{2}\left(\mathbb{R}^{n}\right) \subset L^{2}\left(\mathbb{R}^{n}\right) \cap L^{\frac{2 n}{n-4}}\left(\mathbb{R}^{n}\right),
$$

and with the convexity inequality we get

$$
\lim _{k \rightarrow \infty}\left\|u_{k}\right\|_{q}=0 \forall q \in\left(2, \frac{2 n}{n-4}\right) .
$$

Proposition 5.3. For any $\rho>0$ and $0<\mu<\rho$ the following subadditivity condition holds

$$
J_{\rho, p}<J_{\mu, p}+J_{\sqrt{\rho^{2}-\mu^{2}}, p} .
$$

Proof It is sufficient to prove

$$
J_{\theta \rho, p}<\theta^{2} J_{\rho, p} \forall \rho>0, \theta>1 .
$$

We take $u \in H^{2}\left(\mathbb{R}^{n}\right) \cap B_{L^{2}}(\rho)$ and we set $u_{\theta} \equiv \theta u(x)$. Clearly $\left\|u_{\theta}\right\|_{2}^{2}=\theta^{2} \rho^{2}$ and thus

$$
\begin{array}{r}
J_{\theta \rho, p} \leq \inf _{H^{2}\left(\mathbb{R}^{n}\right) \cap B_{L^{2}}(\rho)}\left(\frac{\theta^{2}}{2}\|\Delta u\|_{2}^{2}-\frac{\theta^{p}}{p} \int_{\mathbb{R}^{n}}|u|^{p} d x\right) \\
<\inf _{H^{2}\left(\mathbb{R}^{n}\right) \cap B_{L^{2}}(\rho)} \theta^{2}\left(\frac{1}{2}\|\Delta u\|_{2}^{2} d x-\frac{1}{p} \int_{\mathbb{R}^{n}}|u|^{p} d x\right)=\theta^{2} J_{\rho, p} .
\end{array}
$$

Proof of Theorem 5.1 Let $u_{k} \in H^{2}\left(\mathbb{R}^{n}\right) \cap B_{L^{2}}\left(\mathbb{R}^{n}\right)$ be a minimising sequence for $J_{\rho, p}$, then due to Proposition 5.1 we have that up to a subsequence we can assume $u_{k} \rightarrow \bar{u}$ in $H^{2}\left(\mathbb{R}^{n}\right)$. Moreover $\bar{u}$ satisfies one of the following conditions:

- $\|\bar{u}\|_{2}=0$;

- $0<\|\bar{u}\|_{2}<\rho$;

- $\|\bar{u}\|_{2}=\rho$.

Notice also that for any sequence $y_{k} \in \mathbb{R}^{n}$ we have that $u_{k}\left(.+y_{k}\right)$ is still a minimizing sequence for $J_{\rho, p}$. This implies that the proof of the Theorem can be concluded provided that we show the existence of a sequence $y_{k} \in \mathbb{R}^{n}$ such that the weak limit of $u_{k}\left(.+y_{k}\right)$ belongs to $B_{L^{2}}(\rho)$.

First step: there exists a sequence $y_{k} \in \mathbb{R}^{n}$ s.t. $u_{k}\left(.+y_{k}\right) \rightarrow \bar{v}$ and $0<\|\bar{v}\|_{2} \leq \rho$ It is sufficient to show that

$$
\sup _{y \in \mathbb{R}^{n}} \int_{Q_{1}(y)}\left|u_{k}\right|^{2} d x \geq \mu>0 .
$$

In fact in this case we can fix $y_{k} \in \mathbb{R}^{n}$ such that

$$
\int_{Q_{1}(0)}\left|u_{k}\left(.+y_{k}\right)\right|^{2} d x \geq \mu>0
$$


and hence, due to the compactness of the embedding $H^{2}\left(Q_{1}(0)\right) \subset L^{2}\left(Q_{1}(0)\right)$, we deduce that the weak limit of the sequence $u_{k}\left(.+y_{k}\right)$ is not the trivial function. It is then sufficient to show (5.6). If by the absurd (5.6) is false then by Proposition 5.2 we get:

and hence

$$
\lim _{k \rightarrow \infty} \int_{\mathbb{R}^{n}}\left|u_{k}\right|^{p} d x=0
$$

$$
J_{\rho, p}=\lim _{k \rightarrow \infty}\left(\frac{1}{2}\left\|\Delta u_{k}\right\|_{2}^{2}-\frac{1}{p} \int_{\mathbb{R}^{n}}\left|u_{k}\right|^{p} d x\right)=\lim _{k \rightarrow \infty} \frac{1}{2}\left\|\Delta u_{k}\right\|_{2}^{2} \geq 0
$$

which is in contradiction with the property $J_{\rho, p}<0$ (see Proposition 5.1).

Second step : if $\bar{v}$ is as in the first step, then $\|\bar{v}\|_{2}=\rho$.

For simplicity we use the notation

$$
v_{k} \equiv u_{k}\left(.+y_{k}\right)
$$

where $y_{k} \in \mathbb{R}^{n}$ is the sequence given in the first step. Notice also that, due to the first step, we have

$$
\|\bar{v}\|_{2}^{2}=\theta^{2} \in\left(0, \rho^{2}\right]
$$

Next assume by the absurd that $0<\theta^{2}<\rho^{2}$. Notice that we have:

$$
\begin{gathered}
\rho^{2}=\left\|v_{k}-\bar{v}\right\|_{2}^{2}+\|\bar{v}\|_{2}^{2}+o(1) \text { i.e. }\left\|v_{k}-\bar{v}\right\|_{2}^{2}=\rho^{2}-\theta^{2}+o(1) \\
\left\|\Delta v_{k}\right\|_{2}^{2}=\left\|\Delta\left(v_{k}-\bar{v}\right)\right\|_{2}^{2}+\|\Delta \bar{v}\|_{2}^{2}+o(1) \\
\left.\left\|v_{k}-\bar{v}\right\|_{p}^{p}+\|\bar{v}\|_{p}^{p}=\left\|v_{k}\right\|_{p}^{p}+o(1) \text { (see the proof of }(3.12)\right)
\end{gathered}
$$

Hence by combining all those facts we deduce:

$$
\begin{gathered}
J_{\theta, p}+J \sqrt{\rho^{2}-\theta^{2}}, p \\
\leq \frac{1}{2}\|\Delta \bar{v}\|_{2}^{2}-\frac{1}{p}\|\bar{v}\|_{p}^{p}+\frac{1}{2} \frac{\rho^{2}-\theta^{2}}{\left\|v_{k}-\bar{v}\right\|_{2}^{2}}\left\|\Delta \bar{v}-\Delta v_{k}\right\|_{2}^{2}-\frac{1}{p}\left\|v_{k}-\bar{v}\right\|_{p}^{p} \frac{\left(\rho^{2}-\theta^{2}\right)^{\frac{p}{2}}}{\left\|v_{k}-\bar{v}\right\|_{2}^{p}} \\
=\frac{1}{2}\|\Delta \bar{v}\|_{2}^{2}-\frac{1}{p}\|\bar{v}\|_{p}^{p}+\frac{1}{2}\left\|\Delta \bar{v}-\Delta v_{k}\right\|_{2}^{2}-\frac{1}{p}\left\|v_{k}-\bar{v}\right\|_{p}^{p}+o(1) \\
=\frac{1}{2}\left\|\Delta v_{k}\right\|_{2}^{2}-\frac{1}{p}\left\|v_{k}\right\|_{p}^{p}+o(1) \rightarrow J_{\rho, p}
\end{gathered}
$$

and this is in contradiction with Proposition 5.3. Hence necessarily we have $\theta^{2}=\rho^{2}$.

\section{REFERENCES}

[1] Alves, M. J.; Carrio, P. C.; Miyagaki, O. H. Soliton solutions to a class of quasilinear elliptic equations on $\mathbb{R}$, Adv. Nonlinear Stud. 7, no. 4, 579-597 (2007)

[2] Bellazzini J., A nonlinear eigenvalue problem for the biharmonic operator in $\mathbb{R}^{N}$, Preprint (2007)

[3] Bonheure D., J. Van Schaftingen, Bound state solutions for a class of nonlinear Schrödinger equations, Rev. Mat. Iberoamericana 24, no. 1, 297-351 (2008).

[4] Cazenave T., Lions P.L., Orbital Stability of Standing Waves for Some Non linear Schrödinger Equations, Commun. Math. Phys. 85, 549-561 (1982)

[5] Chabrowski J., do O M., On some fourth-order semilinear elliptic problems in $R^{N}$, Nonlinear Analysis TMA 49,861-884 (2002)

[6] Deng Y., Li Y., Branches of solutions to semilinear biharmonic equations on $\mathbb{R}^{N}$, Proc. Roy. Soc. Edinburgh Sect. A 136, 733-758 (2006)

[7] Ding W.Y., Ni W.M. On the existence of positive entire solutions of a semilinear elliptic equation, Arch. Rat. Math. Anal. 31, 283-308 (1986). 
[8] Lions P.L., The concentration-compactness principle in the Calculus of Variations. The locally compact case, part 2, Ann. Inst. Henri Poincaré 1, 223-283(1984)

[9] Prinari F., Visciglia N. On a minimization problem involving the critical Sobolev exponent, Adv. Nonl. Studies 7, 551-564 (2007).

[10] Prinari F., Visciglia N. Standing waves for a class of Schrödinger equations with potentials in $L^{\infty}$, to appear on Hokkaido Mathematics Journal.

[11] Wang X., Zeng B. On concentration of positive bound states of Nonlinear Schrödinger Equations with competing potential functions, Siam J. Math. Anal. 28, no. 3, 633-655 (1997). 